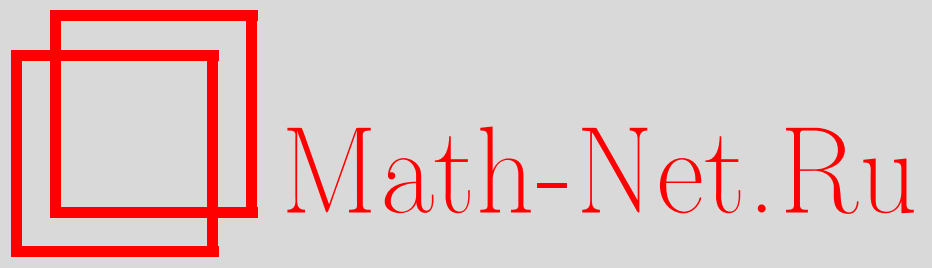

В. Н. Радченко, Эволюционные уравнения с общими стохастическими мерами в гильбертовом пространстве, Теория вероятн. и ее примен., 2014, том 59, выпуск 2, 375-386

DOI: https://doi.org/10.4213/tvp4570

Использование Общероссийского математического портала Math-Net.Ru подразумевает, что вы прочитали и согласны с пользовательским соглашением http://www . mathnet.ru/rus/agreement

Параметры загрузки:

IP: 3.89 .185 .249

26 апреля 2023 г., 17:41:06

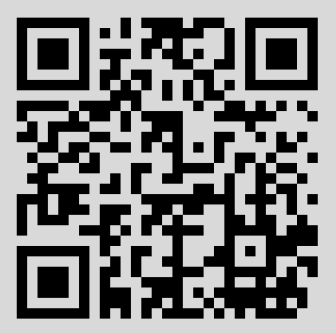




\title{
ЭВОЛЮЦИОННЫЕ УРАВНЕНИЯ С ОБЩИМИ СТОХАСТИЧЕСКИМИ МЕРАМИ В ГИЛЬБЕРТОВОМ ПРОСТРАНСТВЕ ${ }^{1)}$
}

\begin{abstract}
Рассматриваются стохастические эволюционные уравнения в гильбертовом пространстве. На стохастические меры, являющиеся интеграторами в уравнениях, накладывается только условие $\sigma$-аддитивности по вероятности. Определены интегралы от неслучайных функций по стохастическим мерам в гильбертовом пространстве. Доказаны существование и непрерывность мягких решений указанных уравнений.
\end{abstract}

Ключевые слова и фразы: стохастическая мера, стохастическое интегрирование в гильбертовом пространстве, стохастическое эволюционное уравнение, мягкое решение.

1. Введение. В данной работе рассматриваются уравнения

$$
\begin{aligned}
& d X(t)=A X(t)+\sigma(t) d \mu(t), \quad X(0)=X_{0}, \\
& d Y(t)=A Y(t)+B d \mu_{\mathrm{U}}(t), \quad Y(0)=Y_{0},
\end{aligned}
$$

где $A$ - генератор аналитической $C_{0}$-полугруппы $S(t): \mathrm{H} \rightarrow \mathrm{H}, \mathrm{H}$ и $\mathrm{U}$ - действительные сепарабельные гильбертовы пространства, $X(t)$ и $Y(t)$ - искомые Н-значные случайные процессы, $0 \leqslant t \leqslant T$. На стохастические меры $\mu$ (действительнозначную) и $\mu_{U}(\mathrm{U}$-значную) мы накладываем только условия $\sigma$-аддитивности по вероятности. Для этих уравнений мы рассматриваем решения в мягком смысле (см. точные формулировки ниже в (12) и (20)). Исследование мягких решений требует определения Н-значных стохастических интегралов по $\mu$ и $\mu$, что также сделано в работе. При некоторых условиях мы докажем существование и непрерывность этих решений.

Стохастические эволюционные уравнения в гильбертовом пространстве, ведомые действительнозначным винеровским процессом, были рассмотрены в [1]. Уравнение (2), ведомое цилиндрическим процессом Винера $W$, было изучено в $[2$, гл. 4, 5], ведомое процессом Леви - в $[3$, гл. 8, 11, 12]. В [4] ведущим процессом в уравнении был цилиндрический дробный винеровский процесс. Случай Н-значного $W$ и банаховозначного $Y$ был рассмотрен в [5], [6], [7]. Аналогичные уравнения, ведомые банаховозначным броуновским движением, изучены в [8]. Во всех этих случаях, при некоторых предположениях, $Y$ имеет непрерывную модификацию.

В данной работе мы установим существование и непрерывность мягкого решения при более ограничительных условиях, чем в упомянутых работах, однако мы рассматриваем и более широкий класс интеграторов в уравнениях.

В большинстве работ стохастический интеграл определяется с помощью приближения ступенчатыми функциями и некоторых изометрических равенств. Такой подход сложно применить в нашем случае, поскольку значения $\mu$ и $\mu_{U}$ не имеют моментов и это не позволяет найти подходящие нормы для таких равенств. Мы определяем интеграл с помощью некоторых случайных рядов в гильбертовом пространстве.

В п. 2 приводятся основные определения и некоторые предварительные сведения. В п. 3 определяется интеграл от Н-значной функции по действительнозначной

* Киевский национальный университет им. Т. Шевченко, Киев, Украина; е-mail: vradchenko@univ.kiev.ua

1) Работа выполнена при поддержке фонда Александра фон Гумбольдта (грант № 1074615). 
стохастической мере $\mu$ и исследуется уравнение (1). В п. 4 определяется интеграл от операторнозначной функции по $U$-значной стохастической мере $\mu \mathrm{U}$ и рассматривается уравнение (2).

2. Предварительные сведения. Пусть $X$ - произвольное множество, $\mathscr{B}$ $\sigma$-алгебра подмножеств $\mathbf{X},(\Omega, \mathscr{F}, \mathbf{P})$ - полное вероятностное пространство. Через $\mathrm{L}_{0}=\mathrm{L}_{0}(\Omega, \mathscr{F}, \mathbf{P})$ обозначим множество всех случайных величин (точнее говоря, их классов $\mathbf{P}$-эквивалентности). Сходимость в $\mathrm{L}_{0}$ означает сходимость по вероятности.

О п р е д е л е н и е 1 . Стохастической мерой $(\mathrm{CM})$ называется $\sigma$-аддитивное отображение $\mu: \mathscr{B} \rightarrow \mathrm{L}_{0}(\Omega, \mathscr{F}, \mathbf{P})$.

Другими словами, $\mu-$ это векторнозначная мера со значениями в $\mathrm{L}_{0}$. Отметим, что мы не накладываем на $\mu$ никаких условий мартингальности или существования моментов. При необходимости отличать данную СМ от случая гильбертовозначных случайных функций множеств, мы можем говорить, что $\mu-$ действительнозначная CM.

Приведем некоторые примеры. Если $N(t), 0 \leqslant t \leqslant T$, является квадратично интегрируемым мартингалом, то $\mu(\mathrm{A}):=\int_{[0, T]} \mathbf{1}_{\mathrm{A}}(t) d N(t)$ будет $\mathrm{CM}$ на борелевских подмножествах $\mathscr{B}([0, T])$. Аналогичным образом определяет $\mathrm{CM}$ дробное броуновское движение $B^{H}(t)$ при значении параметра Харста $H>1 / 2$ (это следует из [9, неравенство (1.5)]). Частным случаем СМ будут определенные на $\sigma$-алгебре $\alpha$-устойчивые меры с независимыми значениями, подробно рассмотренные в $[10$, гл. 3]. Другие примеры, а также условия того, что приращения случайного процесса с независимыми приращениями порождают случайную меру, можно найти в $[11$, гл. 7,8$]$.

В [12] построен и изучен интеграл вида $\int_{\mathbf{A}} f d \mu$, где $f: \mathbf{X} \rightarrow \mathbf{R}-$ измеримая неслучайная функция, $\mathrm{A} \in \mathscr{R}$. Его конструкция проводится стандартно с использованием приближения простыми функциями. (Аналогичное построение проведено в [11, гл. 7], см. также [13].) В частности, любая ограниченная измеримая функция $f$ будет интегрируемой по любой $\mu$. Для этого интеграла имеет место аналог теоремы Лебега о мажорируемой сходимости (см. [12, следствие 1.2] или [11, предложение 7.1.1]).

В дальнейшем мы предполагаем, что СМ $\mu$ удовлетворяет следующему условию.

У с л о в и е 1 . Существует действительнозначная конечная мера m на $(\mathbf{X}, \mathbf{R})$ со следующим свойством: если для измеримой функции $g: \mathbf{X} \rightarrow \mathbf{R}$ выполняется $\int_{\mathbf{X}} g^{2} d \mathrm{~m}<+\infty$, то $g$ интегрируема по $\mu$ на $\mathbf{X}$.

Данному условию удовлетворяют, например, $\mathrm{CM}$, порожденные $B^{H}(t)$ при $H>$ $3 / 4, \alpha$-устойчивые меры с независимыми значениями на $\mathscr{B}$ при всех $\alpha \in(0,2]$ и $\mathrm{CM}$ с ортогональными значениями.

Определение стохастического интеграла в гильбертовом пространстве будет основано на следующем утверждении.

Лемма 1 (см. [14, лемма 3.3]). Пусть выполнено условие 1 и измеримые функиии $f_{k}: \mathbf{X} \rightarrow \mathbf{R}, k \geqslant 1$, таковы, что

$$
\int_{\mathbf{X}}\left(\sum_{k=1}^{\infty} f_{k}^{2}\right) d \mathrm{~m}<+\infty .
$$

Тогда

$$
\sum_{k=1}^{\infty}\left(\int_{\mathbf{X}} f_{k} d \mu\right)^{2}<+\infty \quad \text { n.н. }
$$

Пусть U - действительное сепарабельное гильбертово пространство, через $\|\cdot\|_{\mathrm{U}}$ мы обозначим норму в $\mathrm{U},(\cdot, \cdot) \mathrm{U}$ обозначает скалярное произведение в $\mathrm{U}$, $\mathrm{L}_{0}(\Omega, \mathscr{F}, \mathbf{P} ; \mathrm{U})$ - это пространство всех U-значных случайных элементов (классов $\mathbf{P}$-эквивалентности), сходимость которых понимается по вероятности. 
О п р е д е л е н и е 2. U-значной стохастической мерой называется $\sigma$-аддитивное отображение $\mu_{\mathrm{U}}: \mathscr{B} \rightarrow \mathrm{L}_{0}(\Omega, \mathscr{F}, \mathbf{P} ; \mathrm{U})$.

Если $\xi_{n}$ - это U-значные случайные элементы такие, что ряд $\sum_{n \geqslant 1} \xi_{n}$ сходится по вероятности безусловно, а $\mathrm{m}_{n}$ - это действительнозначные знакопеременные меры на $\mathscr{B}, \sup _{n, \mathrm{~A}}\left|\mathrm{~m}_{n}(\mathrm{~A})\right| \leqslant 1$, то $\mu_{\mathrm{U}}(\mathrm{A}):=\sum_{n \geqslant 1} \xi_{n} \mathrm{~m}_{n}(\mathrm{~A})$ будет $\mathrm{U}$-значной СМ на $\mathscr{B}$. Это следует из $[15$, теорема 8.6$]$ и $[16$, теорема V.4.2].

Если $M(t), 0 \leqslant t \leqslant T,-\mathrm{U}$-значный квадратично интегрируемый мартингал, то

$$
\mu_{\cup}^{M}(\mathrm{~A}):=\int_{0}^{T}\left(\mathbf{1}_{\mathrm{A}}(s) E\right) d M(s), \quad \mathrm{A} \in \mathscr{B}([0, T]),
$$

будет U-значной CM. Это следует из [3, теорема 8.7(i)]. Здесь $E: \mathrm{U} \rightarrow \mathrm{U}$ обозначает тождественный оператор, и мы используем определение интеграла из [3, п. 8.2].

Следующее утверждение было доказано в лемме работы [17] для действительнозначных CM.

Лемма 2. Пусть $\mu_{\mathrm{U}}-$ это $\mathrm{U}$-значная $C M u a_{n} \in \mathbf{R}, n \geqslant 1$, таковь, ито $\sum_{n=1}^{\infty}\left|a_{n}\right|<\infty$. Пусть $\mathrm{A}_{k n} \in \mathscr{B}, n \geqslant 1,1 \leqslant k \leqslant l_{n}$, таковы, ито для всех $n$ и $k_{1} \neq k_{2}$ множества $\mathrm{A}_{k_{1} n}$ и $\mathrm{A}_{k_{2} n}$ не пересекаются. Тогда

$$
\sum_{n=1}^{\infty} a_{n}^{2} \sum_{k=1}^{l_{n}}\left\|\mu_{\mathrm{U}}\left(\mathrm{A}_{k n}\right)\right\|_{\mathrm{U}}^{2}<\infty \quad \text { n.н. }
$$

Д о к а з а т е л ь с т в о. Предположим, что (4) не выполняется. Обозначим

$$
\Omega_{0}=\left\{\omega \in \Omega: \sum_{n=1}^{\infty} a_{n}^{2} \sum_{k=1}^{l_{n}}\left\|\mu_{\mathrm{U}}\left(\mathrm{A}_{k n}\right)\right\|_{\mathrm{U}}^{2}=+\infty\right\}, \quad \mathbf{P}\left(\Omega_{0}\right)=\alpha_{0}>0 .
$$

Рассмотрим независимые случайные величины $\varepsilon_{k n}, n \geqslant 1,1 \leqslant k \leqslant l_{n}$, определенные на другом вероятностном пространстве $\left(\Omega^{\prime}, \mathscr{F}^{\prime}, \mathbf{P}^{\prime}\right), \mathbf{P}^{\prime}\left(\varepsilon_{k n}=1\right)=\mathbf{P}^{\prime}\left(\varepsilon_{k n}=-1\right)=1 / 2$. Имеем:

$$
\mathbf{E}_{\mathbf{P}^{\prime}}\left\|\sum_{n=1}^{j} a_{n} \sum_{k=1}^{l_{n}} \varepsilon_{k n} \mu_{\mathbf{U}}\left(\mathrm{A}_{k n}\right)\right\|_{\mathbf{U}}^{2}=\sum_{n=1}^{j} a_{n}^{2} \sum_{k=1}^{l_{n}}\left\|\mu_{\mathbf{U}}\left(\mathrm{A}_{k n}\right)\right\|_{\mathbf{U}}^{2}
$$

Значит, для каждого фиксированного $\omega \in \Omega_{0}$ множество случайных величин

$$
\eta_{j}\left(\omega^{\prime}, \omega\right)=\sum_{n=1}^{j} a_{n} \sum_{k=1}^{l_{n}} \varepsilon_{k n}\left(\omega^{\prime}\right) \mu \mathrm{U}\left(\mathrm{A}_{k n}, \omega\right), \quad j \geqslant 1,
$$

не ограничено в $\mathrm{L}_{2}\left(\Omega^{\prime}, \mathscr{F}^{\prime}, \mathbf{P}^{\prime} ; \mathrm{U}\right)$. По теореме V.5.2(a) [16] множество $\eta_{j}\left(\omega^{\prime}, \omega\right), j \geqslant 1$, не ограничено в $\mathrm{L}_{0}\left(\Omega^{\prime}, \mathscr{F}^{\prime}, \mathbf{P}^{\prime} ; \mathrm{U}\right)$. Для некоторого $\alpha_{1}>0$ при каждом $c>0$ найдется $m$ такое, что

$$
\mathbf{P}^{\prime}\left\{\omega^{\prime} \in \Omega^{\prime}: \max _{1 \leqslant j \leqslant m}\left\|\eta_{j}\left(\omega^{\prime}, \omega\right)\right\|_{\mathrm{U}} \geqslant c\right\} \geqslant \alpha_{1} .
$$

Выберем $m$ настолько большим, чтобы (5) выполнялось для всех $\omega \in \Omega_{1} \subset \Omega_{0}$, $\mathbf{P}\left(\Omega_{1}\right) \geqslant \alpha_{0} / 2$. Из неравенства Леви (см. [16, V.2.2(6)]) следует, что для каждого $\omega \in \Omega_{1}$

и мы получаем, что

$$
\mathbf{P}^{\prime}\left\{\omega^{\prime} \in \Omega^{\prime}:\left\|\eta_{m}\left(\omega^{\prime}, \omega\right)\right\|_{\mathrm{U}} \geqslant c\right\} \geqslant \frac{\alpha_{1}}{2}
$$

$$
\mathbf{P} \times \mathbf{P}^{\prime}\left\{\left(\omega, \omega^{\prime}\right) \in \Omega \times \Omega^{\prime}:\left\|\eta_{m}\left(\omega^{\prime}, \omega\right)\right\|_{\mathrm{U}} \geqslant c\right\} \geqslant \frac{\alpha_{0} \alpha_{1}}{4} .
$$

Поэтому можно выбрать и зафиксировать некоторое $\omega^{\prime} \in \Omega^{\prime}$ такое, что

$$
\mathbf{P}\left\{\omega \in \Omega:\left\|\sum_{n=1}^{m} \sum_{k=1}^{l_{n}} a_{n} \varepsilon_{k n}\left(\omega^{\prime}\right) \mu_{\mathbf{U}}\left(\mathrm{A}_{k n}, \omega\right)\right\|_{\mathbf{U}} \geqslant c\right\} \geqslant \frac{\alpha_{0} \alpha_{1}}{4} .
$$


Из алгебры, порожденной набором множеств $\mathrm{A}_{k n}, 1 \leqslant k \leqslant l_{n}, 1 \leqslant m \leqslant n$, мы можем выбрать такие непересекающиеся множества $\mathrm{B}_{r}, 1 \leqslant r \leqslant s$, что

$$
\sum_{n=1}^{m} \sum_{k=1}^{l_{n}} a_{n} \varepsilon_{k n}\left(\omega^{\prime}\right) \mu_{\mathrm{U}}\left(\mathrm{A}_{k n}, \omega\right)=\sum_{r=1}^{s} b_{r} \mu_{\mathrm{U}}\left(\mathrm{B}_{r}, \omega\right),
$$

при этом $\left|b_{r}\right| \leqslant \sum_{n=1}^{\infty}\left|a_{n}\right|$. Из [16, лемма V.4.3(в)] имеем:

$$
\begin{aligned}
\mathbf{P}\left\{\left\|\sum_{r=1}^{s} b_{r} \mu_{\mathrm{U}}\left(\mathrm{B}_{r}\right)\right\|_{\mathrm{U}} \geqslant c\right\} & \leqslant 8 \max _{t_{r}= \pm 1} \mathbf{P}\left\{\left\|\sum_{r=1}^{s} t_{r} \mu_{\mathrm{U}}\left(\mathrm{B}_{r}\right)\right\|_{\mathrm{U}} \geqslant \frac{c}{8 \max \left|b_{r}\right|}\right\} \\
& =8 \mathbf{P}\left\{\left\|\mu_{\mathrm{U}}\left(\mathrm{C}_{1}\right)-\mu_{\mathrm{U}}\left(\mathrm{C}_{2}\right)\right\|_{\mathrm{U}} \geqslant \frac{c}{8 \max \left|b_{r}\right|}\right\}
\end{aligned}
$$

для некоторых множеств $\mathrm{C}_{1}, \mathrm{C}_{2} \in \mathscr{B}$. Напомним, что $c$ в (5) можно взять сколь угодно большим. Из (6) и (7) мы получаем противоречие с ограниченностью множества значений U-значной CM [18, теорема]. Лемма 2 доказана.

\section{3. Уравнения и интегралы с действительнозначными СМ.}

3.1. Интегралы по действительнозначным СМ. Всюду в этом пункте $\mu-$ действительнозначная СМ, для которой выполняется условие 1 .

Пусть $\mathrm{H}$ - действительное сепарабельное гильбертово пространство; $\|\cdot\|$ - норма в $\mathrm{H} ;(\cdot, \cdot)$ - скалярное произведение в $\mathrm{H} ; e_{j}, j \geqslant 1$, - ортонормированный базис в $\mathrm{H}$; $f: \mathrm{X} \rightarrow \mathrm{H}$ - измеримая функция,

$$
f(x)=\sum_{j=1}^{\infty} e_{j} f_{j}(x), \quad f_{j}: \mathbf{X} \rightarrow \mathbf{R}, \quad \sum_{j=1}^{\infty} f_{j}^{2}(x)=\|f(x)\|^{2} .
$$

О п р е д е л е н и е 3 . Пусть измеримая функция $f: \mathrm{X} \rightarrow \mathrm{H}$ такова, что

$$
\int_{\mathbf{X}}\|f(x)\|^{2} d \mathrm{~m}(x)<+\infty
$$

Тогда положим

$$
\int_{\mathbf{X}} f d \mu:=\sum_{j=1}^{\infty} e_{j} \int_{\mathbf{X}} f_{j} d \mu
$$

где ряд (9) сходится безусловно п.н. в Н.

Сходимость ряда (9) следует из леммы 1 и (8). Легко проверить, что $\int_{\mathbf{X}} c \mathbf{1}_{\mathrm{A}} d \mu=$ $c \mu(\mathrm{A}), c \in \mathrm{H}, \mathrm{A} \in \mathscr{B}$.

Покажем, что значение интеграла не зависит от выбора базиса. Пусть $u_{r}, r \geqslant 1,-$ другой ортонормированный базис, $c_{r j}=\left(u_{r}, e_{j}\right), f_{r}^{\prime}=\left(f, u_{r}\right)$. Имеем

$$
\begin{aligned}
\sum_{r=1}^{\infty} u_{r} \int_{\mathbf{X}} f_{r}^{\prime} d \mu & =\sum_{r=1}^{\infty} \sum_{j=1}^{\infty} c_{r j} e_{j} \int_{\mathbf{X}} f_{r}^{\prime} d \mu=\sum_{j=1}^{\infty} e_{j} \sum_{r=1}^{\infty} c_{r j} \int_{\mathbf{X}} f_{r}^{\prime} d \mu \\
& \stackrel{(*)}{=} \sum_{j=1}^{\infty} e_{j} \int_{\mathbf{X}}\left(\sum_{r=1}^{\infty} c_{r j} f_{r}^{\prime}\right) d \mu=\sum_{j=1}^{\infty} e_{j} \int_{\mathbf{X}} f_{j} d \mu .
\end{aligned}
$$

Для обоснования равенства $(*)$ мы можем использовать оценку

$$
\left(\sum_{r=1}^{\infty}\left|c_{r j} f_{r}^{\prime}\right|\right)^{2} \leqslant \sum_{r=1}^{\infty} c_{r j}^{2} \sum_{r=1}^{\infty} f_{r}^{\prime 2}=\|f(x)\|^{2} .
$$

По условию 1 и (8), функция $\sum_{r=1}^{\infty}\left|c_{r j} f_{r}^{\prime}\right|$ интегрируема по $\mu$, и $(*)$ следует из теоремы о доминируемой сходимости [11, предложение 7.1.1]. 
Теорема 1. Если измеримье $f^{(n)}: \mathrm{X} \rightarrow \mathrm{H}, n \geqslant 1$, таковы, ито

$$
\int_{\mathbf{X}}\left\|f^{(n)}(x)\right\|^{2} d \mathrm{~m}(x) \rightarrow 0
$$

mo

$$
\int_{\mathbf{X}} f^{(n)} d \mu \stackrel{\mathbf{P}}{\longrightarrow} 0, \quad n \rightarrow \infty .
$$

Д о к а з а т е ль с т в о. Если утверждение теоремы неверно, то найдутся $f^{(n)}: \mathrm{X} \rightarrow \mathrm{H}$ такие, что

$$
\int_{\mathbf{X}}\left\|f^{(n)}(x)\right\|^{2} d \mathbf{m}(x)<2^{-n}, \quad \int_{\mathbf{X}} f^{(n)} d \mu \stackrel{\mathbf{P}}{\nrightarrow} 0 .
$$

Для $f_{j}^{(n)}=\left(f^{(n)}, e_{j}\right)$ имеем

$$
\sum_{n=1}^{\infty} \int_{\mathbf{X}}\left\|f^{(n)}(x)\right\|^{2} d \mathrm{~m}(x)=\sum_{n=1}^{\infty} \sum_{j=1}^{\infty} \int_{\mathbf{X}} f_{j}^{(n)}(x)^{2} d \mathrm{~m}(x)<+\infty,
$$

и из леммы 1 получаем, что

$$
\sum_{n=1}^{\infty}\left\|\int_{\mathbf{X}} f^{(n)} d \mu\right\|^{2}=\sum_{n=1}^{\infty} \sum_{j=1}^{\infty}\left(\int_{\mathbf{X}} f_{j}^{(n)}(x) d \mu(x)\right)^{2}<+\infty \text { п.н. }
$$

Это противоречит предположению $\int_{\mathbf{X}} f^{(n)} d \mu \stackrel{\mathrm{P}}{\nrightarrow} 0$. Теорема доказана.

Далее мы рассмотрим случай $\mathrm{X}=[0, T]$. Будем использовать обозначения

$$
\begin{array}{cl}
d_{k n}=k \cdot 2^{-n} T, \quad n \geqslant 0, & 0 \leqslant k \leqslant 2^{n}, \\
\Delta_{k n}=\left(d_{(k-1) n}, d_{k n}\right], & 1 \leqslant k \leqslant 2^{n} .
\end{array}
$$

Следующая лемма является ключевой в доказательстве непрерывности траекторий интеграла.

Лемма 3. Пусть $\mathscr{B}-$ борелевская $\sigma$-алгебра в $[0, T], \mathrm{A} \in \mathscr{B}, Z-$ произвольное множество и функция $q(z, s): Z \times[0, T] \rightarrow \mathrm{H}$ такова, ито все траектории $q(z, \cdot)$ непрерывны на $[0, T]$. Обозначим

$$
q_{n}(z, s)=q(z, 0) \mathbf{1}_{\{0\}}(s)+\sum_{1 \leqslant k \leqslant 2^{n}} q\left(z, d_{(k-1) n}\right) \mathbf{1}_{\Delta_{k n}}(s) .
$$

Тогда случайная функция

$$
\eta(z)=\int_{\mathrm{A}} q(z, s) d \mu(s), \quad z \in Z
$$

имеет модификачию

$$
\widetilde{\eta}(z)=\int_{\mathrm{A}} q_{0}(z, s) d \mu(s)+\sum_{n \geqslant 1}\left(\int_{\mathrm{A}} q_{n}(z, s) d \mu(s)-\int_{\mathrm{A}} q_{n-1}(z, s) d \mu(s)\right)
$$

такую, что для всех $\beta>0, \omega \in \Omega, z \in Z$

$$
\begin{aligned}
\|\widetilde{\eta}(z)\| \leqslant & \|q(z, 0) \mu(\mathrm{A})\|+\left\{\sum_{n \geqslant 1} 2^{n \beta} \sum_{1 \leqslant k \leqslant 2^{n}}\left\|q\left(z, d_{k n}\right)-q\left(z, d_{(k-1) n}\right)\right\|^{2}\right\}^{1 / 2} \\
& \times\left\{\sum_{n \geqslant 1} 2^{-n \beta} \sum_{1 \leqslant k \leqslant 2^{n}}\left|\mu\left(\Delta_{k n} \cap \mathrm{A}\right)\right|^{2}\right\}^{1 / 2} .
\end{aligned}
$$


Д о к а з а т е л ь с т в о. Из непрерывности $q(z, \cdot)$ и теоремы 1 получаем, что

$$
\int_{\mathrm{A}} q_{n}(z, s) d \mu(s) \stackrel{\mathbf{P}}{\rightarrow} \int_{\mathrm{A}} q(z, s) d \mu(s), \quad n \rightarrow \infty,
$$

для каждого $z$. Значит, $\widetilde{\eta}(z)$ является модификацией $\eta(z)$. Для $\beta>0$ имеем

$$
\begin{aligned}
\sum_{n \geqslant 1} & \left\|\int_{\mathrm{A}} q_{n}(z, s) d \mu(s)-\int_{\mathrm{A}} q_{n-1}(z, s) d \mu(s)\right\| \\
\leqslant & \sum_{n \geqslant 1} \sum_{1 \leqslant k \leqslant 2^{n}}\left\|q\left(z, d_{(k-1) n}\right)-q\left(z, d_{\left(k^{\prime}-1\right)(n-1)}\right)\right\|\left|\mu\left(\Delta_{k n} \cap \mathrm{A}\right)\right| \\
\leqslant & \left\{\sum_{n \geqslant 1} \sum_{1 \leqslant k \leqslant 2^{n}} 2^{n \beta}\left\|q\left(z, d_{(k-1) n}\right)-q\left(z, d_{\left(k^{\prime}-1\right)(n-1)}\right)\right\|^{2}\right\}^{1 / 2} \\
& \times\left\{\sum_{n \geqslant 1} \sum_{1 \leqslant k \leqslant 2^{n}} 2^{-n \beta}\left|\mu\left(\Delta_{k n} \cap \mathrm{A}\right)\right|^{2}\right\}^{1 / 2} .
\end{aligned}
$$

(Номер $k^{\prime}$ выбран так, что $\Delta_{k n} \subset \Delta_{k^{\prime}(n-1)}$.) Лемма 3 доказана.

Отметим, что по лемме 2 для каждого $\beta>0$

$$
\sum_{n \geqslant 1} \sum_{1 \leqslant k \leqslant 2^{n}} 2^{-n \beta}\left|\mu\left(\Delta_{k n} \cap \mathrm{A}\right)\right|^{2}<+\infty \quad \text { п.н. }
$$

3.2. Эволюционные уравнения, ведомые действительнозначными СМ. В этом пункте $\mathscr{B}-$ борелевская $\sigma$-алгебра в $\mathrm{X}=[0, T], \sigma(t):[0, T] \rightarrow \mathrm{H}$ - измеримая функция, $X_{0} \in \mathrm{H}$, и мы рассмотрим мягкое решение уравнения (1)

$$
X(t)=S(t) X_{0}+\int_{(0, t]} S(t-s) \sigma(s) d \mu(s), \quad 0<t \leqslant T .
$$

Для существования интеграла в (12) достаточно выполнения условия

$$
\int_{[0, T]}\|\sigma(s)\|^{2} d \mathrm{~m}(s)<+\infty .
$$

У с л о в и е 2. Функция $\sigma$ представляется в виде $\sigma(s)=\widetilde{\sigma}(s) \mathbf{1}_{\mathrm{B}}(s)$, где $\mathrm{B} \in \mathscr{B}$, и для некоторого $1 / 2<\varepsilon \leqslant 1$

$$
\tilde{\sigma}(s) \in D\left(A^{\varepsilon}\right), \quad \sup _{s \in[0, T]}\left\|A^{\varepsilon} \widetilde{\sigma}(s)\right\|<+\infty, \quad\|\widetilde{\sigma}(s)-\widetilde{\sigma}(u)\| \leqslant C|s-u|^{\varepsilon} .
$$

У с л о в и е 3 . Для множества В из условия 2 процесс $\mu((0, s] \cap \mathrm{B}), 0 \leqslant s \leqslant T$, имеет непрерывную модификацию.

Всюду в неравенствах $C$ обозначает положительную константу, конкретное значение которой несущественно. Через $A^{\varepsilon}$ мы обозначаем степень соответствующего оператора.

Теорема 2. Пусть выполнены условия 2 и 3 . Тогда прочесс $X(t)$, определенныци в (12), имеет непрерывную модификацию.

Д о к а з а т е л ь с т в о. Имеем

$$
\begin{aligned}
\int_{(0, t]} S(t-s) \sigma(s) d \mu(s)= & \int_{(0, T] \cap \mathrm{B}} S((t-s) \vee 0) \widetilde{\sigma}(s) d \mu(s) \\
& -\int_{(t, T] \cap \mathrm{B}} \tilde{\sigma}(s) d \mu(s)=: \eta_{1}(t)-\eta_{2}(t) .
\end{aligned}
$$

Для интегралов $\eta_{1}(t)$ и $\eta_{2}(t)$ мы выберем модификации, определенные в (10). 
Возьмем $0 \leqslant t_{1}<t_{2}$ и рассмотрим пределы при $\left|t_{2}-t_{1}\right| \rightarrow 0$. Из леммы 3 имеем, что

$$
\begin{aligned}
& \left\|\eta_{1}\left(t_{2}\right)-\eta_{1}\left(t_{1}\right)\right\|=\left\|\int_{(0, T] \cap \mathrm{B}}\left(S\left(\left(t_{2}-s\right) \vee 0\right)-S\left(\left(t_{1}-s\right) \vee 0\right)\right) \widetilde{\sigma}(s) d \mu(s)\right\| \\
& \leqslant\left\|\left(S\left(t_{2}\right)-S\left(t_{1}\right)\right) \widetilde{\sigma}(0)\right\||\mu((0, T] \cap \mathrm{B})| \\
& \quad+\left\{\sum_{n \geqslant 1} 2^{n \beta} \sum_{1 \leqslant k \leqslant 2^{n}} \|\left(S\left(\left(t_{2}-d_{k n}\right) \vee 0\right)-S\left(\left(t_{1}-d_{k n}\right) \vee 0\right)\right) \widetilde{\sigma}\left(d_{k n}\right)\right. \\
& \left.\quad-\left(S\left(\left(t_{2}-d_{(k-1) n}\right) \vee 0\right)-S\left(\left(t_{1}-d_{(k-1) n}\right) \vee 0\right)\right) \widetilde{\sigma}\left(d_{(k-1) n}\right) \|^{2}\right\}^{1 / 2} \\
& \quad \times\left\{\sum_{n \geqslant 1} 2^{-n \beta} \sum_{1 \leqslant k \leqslant 2^{n}}\left|\mu\left(\Delta_{k n} \cap(0, T] \cap \mathrm{B}\right)\right|^{2}\right\}^{1 / 2}=: I_{1}+I_{2}^{1 / 2} \times I_{3}^{1 / 2} .
\end{aligned}
$$

Очевидно, что $I_{1} \rightarrow 0$. Из [19, теорема $\left.2.6 .13(\mathrm{~d})\right]$ и стандартных свойств полугрупп имеем, что

$$
\|S(s) h-S(t) h\| \leqslant C|s-t|^{\varepsilon}\left\|A^{\varepsilon} h\right\|, \quad s, t \in[0, T], \quad h \in D\left(A^{\varepsilon}\right) .
$$

Используя эту оценку и условие 2 , для $0<\beta<2 \varepsilon-1$ в $I_{2}$ получим

$$
\begin{aligned}
\sum_{n \geqslant 1} 2^{n \beta} \sum_{1 \leqslant k \leqslant 2^{n}}\left(\left\|S\left(\left(t_{2}-d_{k n}\right) \vee 0\right)\left[\widetilde{\sigma}\left(d_{k n}\right)-\widetilde{\sigma}\left(d_{(k-1) n}\right)\right]\right\|^{2}\right. \\
\left.\quad+\left\|\left[S\left(\left(t_{2}-d_{k n}\right) \vee 0\right)-S\left(\left(t_{2}-d_{(k-1) n}\right) \vee 0\right)\right] \widetilde{\sigma}\left(d_{(k-1) n}\right)\right\|^{2}\right) \\
\leqslant C \sum_{n \geqslant 1} 2^{n \beta} 2^{n(1-2 \varepsilon)}<+\infty .
\end{aligned}
$$

Аналогичные оценки имеют место для слагаемых $S\left(\left(t_{1}-\cdot\right) \vee 0\right)$. Условие 2 и неравенство (13) дают нам оценку сверху для $I_{2}$, каждое слагаемое в $I_{2}$ стремится к 0 , и поэтому $I_{2} \rightarrow 0$. Напомним, что $I_{3}<\infty$ п.н. по лемме 2. Значит, $\eta_{1}$ непрерывен.

Далее из леммы 3 получаем

$$
\begin{aligned}
\left\|\eta_{2}\left(t_{2}\right)-\eta_{2}\left(t_{1}\right)\right\|= & \left\|\int_{\left(t_{1}, t_{2}\right] \cap \mathrm{B}} \tilde{\sigma}(s) d \mu(s)\right\| \leqslant\|\widetilde{\sigma}(0)\|\left|\mu\left(\left(t_{1}, t_{2}\right] \cap \mathrm{B}\right)\right| \\
& +\left\{\sum_{n \geqslant 1} 2^{n \beta} \sum_{1 \leqslant k \leqslant 2^{n}}\left\|\widetilde{\sigma}\left(d_{k n}\right)-\widetilde{\sigma}\left(d_{(k-1) n}\right)\right\|^{2}\right\}^{1 / 2} \\
& \times\left\{\sum_{n \geqslant 1} 2^{-n \beta} \sum_{1 \leqslant k \leqslant 2^{n}}\left|\mu\left(\Delta_{k n} \cap\left(t_{1}, t_{2}\right] \cap \mathrm{B}\right)\right|^{2}\right\}^{1 / 2} \\
=: & J_{1}+J_{2}^{1 / 2} \times J_{3}^{1 / 2} .
\end{aligned}
$$

Имеем

$$
\begin{aligned}
J_{3} \leqslant & \sum_{n \geqslant 1} 2^{-n \beta} \sum_{k: \Delta_{k n} \subset\left(t_{1}, t_{2}\right]}\left|\mu\left(\Delta_{k n} \cap \mathrm{B}\right)\right|^{2}+\sum_{n \geqslant 1} 2^{-n \beta}\left|\mu\left(\Delta_{k^{\prime} n} \cap\left(t_{1}, t_{2}\right] \cap \mathrm{B}\right)\right|^{2} \\
& +\sum_{n \geqslant 1} 2^{-n \beta}\left|\mu\left(\Delta_{k^{\prime \prime} n} \cap\left(t_{1}, t_{2}\right] \cap \mathrm{B}\right)\right|^{2} \\
= & : J_{31}+J_{32}+J_{33}, \quad t_{1} \in \Delta_{k^{\prime} n}, \quad t_{2} \in \Delta_{k^{\prime \prime} n} .
\end{aligned}
$$


Из условия 3 следует, что $J_{1}, J_{32}, J_{33} \rightarrow 0$, а $J_{31} \rightarrow 0$ как остатки сходящихся рядов (11). Используя условие 2 , для $0<\beta<2 \varepsilon-1$ получаем $J_{2}<\infty$. Поэтому $\eta_{2}$ непрерывен. Теорема 2 доказана.

Отметим, что условие $\widetilde{\sigma}(s) \in D\left(A^{\varepsilon}\right)$ также накладывалось в замечании 4.3 и теореме 2.1 [1], устанавливающих существование непрерывного решения неоднородного эволюционного уравнения, ведомого винеровским процессом.

\section{4. Уравнения и интегралы с U-значными CM.}

4.1. Интегралы по U-значным CM. Всюду в этом пункте $\mu_{\mathrm{U}}-$ это $\mathrm{U}$ значная $\mathrm{CM}$ и $B(x): \mathrm{X} \rightarrow L(\mathrm{U}, \mathrm{H})-$ функция со значениями в множестве непрерывных линейных операторов из $\mathrm{U}$ в $\mathrm{H}$. Предполагаем, что для каждого $\varphi \in \mathrm{U}$ функция $B(x) \varphi: \mathrm{X} \rightarrow \mathrm{H}$ измерима.

Пусть $\varphi_{i}, i \geqslant 1$, 一 ортонормированный базис в U. Очевидно, что $\mu_{i}(\mathrm{~A})=$ $\left(\mu_{\mathrm{U}}(\mathrm{A}), \varphi_{i}\right) \mathrm{U}$ будут действительнозначными $\mathrm{CM}$,

$$
\mu_{\mathrm{U}}(\mathrm{A})=\sum_{i=1}^{\infty} \mu_{i}(\mathrm{~A}) \varphi_{i} .
$$

У с л о в и е 4. Для каждого $i \geqslant 1$ существует действительнозначная конечная мера $\mathrm{m}_{i}$ на $(\mathrm{X}, \mathscr{B})$ со следующим свойством: если измеримая функция $h: \mathrm{X} \rightarrow \mathbf{R}$ такова, что $\int_{\mathbf{X}} h^{2} d \mathrm{~m}_{i}<+\infty$, то $h$ интегрируема по $\mu_{i}$ на $\mathbf{X}$.

О п р е д е л е н и е 4. Пусть выполнено условие 4 и

$$
\int_{\mathbf{X}}\left\|B(x) \varphi_{i}\right\|^{2} d \mathrm{~m}_{i}(x)<+\infty, \quad i \geqslant 1
$$

Тогда положим

$$
\int_{\mathbf{X}} B(x) d \mu_{\mathbf{U}}(x):=\sum_{i=1}^{\infty} \int_{\mathbf{X}}\left(B(x) \varphi_{i}\right) d \mu_{i}(x),
$$

если ряд сходится по вероятности безусловно в U, и сумма не зависит от выбора ортонормированного базиса.

Интегралы по $\mu_{i}$ здесь взяты по определению 3. Легко видеть, что

$$
\int_{\mathbf{X}}{ }^{1} \mathrm{~A}^{B(x) d \mu_{\mathbf{U}}}=\int_{\mathbf{A}} B(x) d \mu_{\mathbf{U}}, \quad \mathbf{A} \in \mathscr{B} .
$$

Всюду ниже мы предполагаем, что для $\mu_{U}$ выполняется условие 4. Также мы будем использовать операторную норму Гильберта-Шмидта

$$
\|B(x)\|_{\mathrm{HS}}^{2}=\sum_{i=1}^{\infty}\left\|B(x) \varphi_{i}\right\|^{2} .
$$

П р и м е р 1 . Для $B(x)=\mathbf{1}_{\mathrm{A}}(x) B$ имеем

$$
\begin{aligned}
\int_{\mathbf{X}}{ }^{1} A B d \mu_{U} & =\sum_{i=1}^{\infty} \int_{\mathbf{X}}{ }_{1}{ }_{A}\left(B \varphi_{i}\right) d\left(\mu_{\mathbf{U}}, \varphi_{i}\right)_{\mathbf{U}}=\sum_{i=1}^{\infty}\left(B \varphi_{i}\right)\left(\mu_{\mathbf{U}}(\mathrm{A}), \varphi_{i}\right)_{\mathrm{U}} \\
& =B \sum_{i=1}^{\infty} \varphi_{i}\left(\mu_{\mathbf{U}}(\mathrm{A}), \varphi_{i}\right)_{\mathbf{U}}=B \mu_{\mathbf{U}}(\mathrm{A}) .
\end{aligned}
$$

П р и м е р 2. Для U-значного квадратично интегрируемого мартингала Леви $M$ сравним интеграл по $M$, построенный в $\left[3\right.$, гл. 8], и наш интеграл по $\mu_{\mathrm{U}}^{M}$ (см. (3)).

Пусть $M(t), 0 \leqslant t \leqslant T$, - U-значный стохастически непрерывный процесс с независимыми приращениями, у которого распределение $M(t)-M(s)$ зависит только от $t-s, \mathbf{E} M(t)=0, \mathbf{E} M(t)^{2}<+\infty$. Его предсказуемая характеристическая вариация равна $\langle M, M\rangle_{s}=s \operatorname{Tr} Q$ для некоторого неотрицательного ядерного оператора $Q: \mathrm{U} \rightarrow$ 
$\mathrm{U}$ (см. [3, п. 8.6.1]). Для случайной неупреждающей операторной функции $\Psi(s, \omega)$ в $[3]$ определен интеграл $I_{T}^{M}(\Psi)=\int_{0}^{T} \Psi(s, \omega) d M(s)$, если

$$
\|\Psi\|_{M, T}^{2}=\mathbf{E} \int_{0}^{T}\left\|\Psi(s) Q^{1 / 2}\right\|_{\mathrm{HS}}^{2} d\langle M, M\rangle_{s}<+\infty
$$

(см. [3, (8.6) и теорема 8.7]). При этом

$$
\mathbf{E}\left|I_{T}^{M}(\Psi)\right|^{2}=\|\Psi\|_{M, T}^{2} .
$$

$\mathrm{K}$ порожденной данным мартингалом Леви U-значной $\mathrm{CM} \mu_{\mathrm{U}}^{M}$ можно применить наше определение 4. Имеем, что $\left(M(s), \varphi_{i}\right) \mathrm{U}$ - это действительнозначный процесс Леви, условие 4 выполняется для $\mu_{i}^{M}(\mathrm{~A})=\left(\mu_{\mathrm{U}}^{M}(\mathrm{~A}), \varphi_{i}\right) \cup$ и $\mathrm{m}_{i}(d s)=d s$ (мы можем использовать (15) и (16) для $\mathrm{U}=\mathrm{H}=\mathbf{R})$.

Пусть $B(s)$ - неслучайная операторная функция, интегрируемая по $d M(s)$ в смысле [3]. Пусть $\varphi_{i}, i \geqslant 1,-$ базис из собственных векторов оператора $Q$. Рассмотрим последовательность операторов

$$
B_{n}(s) u=\sum_{i=1}^{n}\left(u, \varphi_{i}\right) \mathrm{U}^{B(s) \varphi_{i},} \quad u \in \mathrm{U}, \quad n \geqslant 1 .
$$

Тогда

$$
\sum_{i=1}^{n} \int_{[0, T]}\left(B(s) \varphi_{i}\right) d \mu_{i}^{M}(s)=\int_{[0, T]} B_{n}(s) d M(s), \quad\left\|B_{n}(s) Q^{1 / 2}\right\|_{\mathrm{HS}} \leqslant\left\|B(s) Q^{1 / 2}\right\|_{\mathrm{HS}} .
$$

Из (15), (16) и теоремы о мажорируемой сходимости следует, что

$$
\int_{[0, T]} B_{n}(s) d M(s) \rightarrow \int_{[0, T]} B(s) d M(s)
$$

в $L_{2}$-норме. Значит, в этом случае для данных $\varphi_{i}$ ряд (14) сходится к $\int_{[0, T]} B(s) d M(s)$. (Отметим, что для интеграла по $Q$-винеровскому процессу $W$ сходимость (14) следует из результатов $[2$, п. 4.3.2].)

Для указанной $\mu_{\bigcup}^{M}$ мы не можем гарантировать сходимость (14) для любого базиса, но если оба интеграла $\int_{[0, T]} B(s) d M(s)$ и $\int_{[0, T]} B(s) d \mu_{U}^{M}(s)$ существуют, то они равны.

Определение 4 аналогично определению интеграла по цилиндрическому дробному броуновскому движению в [4]. Интеграл в [4] определен как сумма (14) для $\mu_{i}$, порожденных независимыми действительнозначными дробными броуновскими движениями, и значение интеграла может зависеть от ортонормированного базиса.

Далее мы дадим достаточное условие существования нашего интеграла в случае $\mathrm{X} \subset[0, T]$.

Теорема 3. Пусть $\mathscr{B}-$ борелевская $\sigma$-алгебра в $[0, T], \mathrm{A} \in \mathscr{B}, B(s)$ - операторы Гильберта-Шмидта, $\mathrm{H}$-значные функции $B(s) \varphi_{i}, 0 \leqslant s \leqslant T$, непрерывны и

$$
\sum_{n \geqslant 1} 2^{n \beta} \sum_{1 \leqslant k \leqslant 2^{n}}\left\|B\left(d_{k n}\right)-B\left(d_{(k-1) n}\right)\right\|_{\mathrm{HS}}^{2}<+\infty
$$

для некоторого $\beta>0$. Тогда определен $\int_{\mathrm{A}} B(s) d \mu_{\mathrm{U}}(s)$.

Д о к а з а т е ль с т в о. Сначала мы покажем, что сходится ряд (14). Из леммы 3 следует, что

$$
\sum_{i=1}^{\infty}\left\|\int_{\mathrm{A}} B(s) \varphi_{i} d \mu_{i}(s)\right\| \leqslant \sum_{i=1}^{\infty}\left(\left\|\left(B(0) \varphi_{i}\right) \mu_{i}(\mathrm{~A})\right\|\right.
$$




$$
\begin{aligned}
& +\left\{\sum_{n \geqslant 1} 2^{n \beta} \sum_{1 \leqslant k \leqslant 2^{n}}\left\|B\left(d_{k n}\right) \varphi_{i}-B\left(d_{(k-1) n}\right) \varphi_{i}\right\|^{2}\right\}^{1 / 2} \\
& \left.\times\left\{\sum_{n \geqslant 1} 2^{-n \beta} \sum_{1 \leqslant k \leqslant 2^{n}}\left|\mu_{i}\left(\Delta_{k n} \cap \mathrm{A}\right)\right|^{2}\right\}^{1 / 2}\right) .
\end{aligned}
$$

Для первого слагаемого в правой части (18) имеем

$$
\begin{aligned}
\sum_{i=1}^{\infty}\left\|\left(B(0) \varphi_{i}\right) \mu_{i}(\mathrm{~A})\right\| & \leqslant\left[\sum_{i=1}^{\infty}\left\|B(0) \varphi_{i}\right\|^{2}\right]^{1 / 2}\left[\sum_{i=1}^{\infty}\left|\mu_{i}(\mathrm{~A})\right|^{2}\right]^{1 / 2} \\
& =\|B(0)\|_{\mathrm{HS}}\left\|\mu_{\mathrm{U}}(\mathrm{A})\right\|_{\mathrm{U}}<+\infty
\end{aligned}
$$

Для остальных слагаемых в (18) мы используем неравенство $2 \sqrt{a b} \leqslant a+b$ и получим

$$
\begin{aligned}
& \sum_{i=1}^{\infty} \sum_{n \geqslant 1} 2^{n \beta} \sum_{1 \leqslant k \leqslant 2^{n}}\left\|B\left(d_{k n}\right) \varphi_{i}-B\left(d_{(k-1) n}\right) \varphi_{i}\right\|^{2} \\
& =\sum_{n \geqslant 1} 2^{n \beta} \sum_{1 \leqslant k \leqslant 2^{n}}\left\|B\left(d_{k n}\right)-B\left(d_{(k-1) n}\right)\right\|_{\mathrm{HS}}^{2} \stackrel{(17)}{<}+\infty, \\
& \sum_{i=1}^{\infty} \sum_{n \geqslant 1} 2^{-n \beta} \sum_{1 \leqslant k \leqslant 2^{n}}\left|\mu_{i}\left(\Delta_{k n} \cap \mathrm{A}\right)\right|^{2} \\
& =\sum_{n \geqslant 1} 2^{-n \beta} \sum_{1 \leqslant k \leqslant 2^{n}}\left\|\mu_{\mathrm{U}}\left(\Delta_{k n} \cap \mathrm{A}\right)\right\|_{\mathrm{U}}^{2 \stackrel{(4)}{<}+\infty .}
\end{aligned}
$$

Значит, сумма (18) конечна.

Далее мы покажем, что сумма (14) не зависит от выбора базиса. Имеем

$$
\begin{aligned}
& \sum_{i=1}^{\infty} \int_{\mathrm{A}}\left(B(x) \varphi_{i}\right) d \mu_{i}(x) \stackrel{(10)}{=} \sum_{i=1}^{\infty}\left(\left(B(0) \varphi_{i}\right) \mu_{i}(\mathrm{~A})\right. \\
& \left.\quad+\sum_{n \geqslant 1} \sum_{1 \leqslant k \leqslant 2^{n}}\left(B\left(d_{(k-1) n}\right) \varphi_{i}-B\left(d_{\left(k^{\prime}-1\right)(n-1)}\right) \varphi_{i}\right) \mu_{i}\left(\Delta_{k n} \cap \mathrm{A}\right)\right) \\
& \quad=B(0) \sum_{i=1}^{\infty} \varphi_{i} \mu_{i}(\mathrm{~A})+\sum_{n \geqslant 1} \sum_{1 \leqslant k \leqslant 2^{n}}\left(B\left(d_{(k-1) n}\right)-B\left(d_{\left(k^{\prime}-1\right)(n-1)}\right)\right) \sum_{i=1}^{\infty} \varphi_{i} \mu_{i}\left(\Delta_{k n} \cap \mathrm{A}\right) \\
& \quad=B(0) \mu_{\mathrm{U}}(\mathrm{A})+\sum_{n \geqslant 1} \sum_{1 \leqslant k \leqslant 2^{n}}\left(B\left(d_{(k-1) n}\right)-B\left(d_{\left(k^{\prime}-1\right)(n-1)}\right)\right) \mu_{\mathrm{U}}\left(\Delta_{k n} \cap \mathrm{A}\right) .
\end{aligned}
$$

Здесь мы можем поменять порядок слагаемых, поскольку сумма (18) конечна и ряд сходится абсолютно. (Номер $k^{\prime}$ взят так, что $\Delta_{k n} \subset \Delta_{k^{\prime}(n-1)}$.) Теорема 3 доказана.

3 а м е ч а н и е. Если $B(0)$ - оператор Гильберта-Шмидта, то неравенство (17) эквивалентно следующему условию: все действительнозначные функции $b_{i j}(s)=\left(B(s) \varphi_{i}, e_{j}\right)$ принадлежат пространству Бесова $B_{22}^{(\beta+1) / 2}([0, T])$ и

$$
\sum_{i \geqslant 1} \sum_{j \geqslant 1}\left\|b_{i j}(s)\right\|_{B_{22}^{(\beta+1) / 2}([0, T])}^{2}<+\infty .
$$

Это следует из [20, теорема 1.1].

Также для справедливости (17) достаточно выполнения условия Гёльдера

$$
\|B(s)-B(t)\|_{\mathrm{HS}} \leqslant C|s-t|^{\varepsilon}, \quad \varepsilon>\frac{\beta+1}{2} .
$$

Следующий пример показывает, что мы не можем убрать условие (17) в теореме 3. 
П р и м е р 3. Возьмем $\mathrm{X}=[0, \pi], \mathrm{U}=\mathrm{H}$ и положим

$$
B(s) \varphi_{i}=\sum_{j=1}^{\infty} \varphi_{j} \frac{1}{(i j)^{3 / 4}} \sin i s, \quad\left\|B(s) \varphi_{i}\right\|^{2} \leqslant 3 i^{-3 / 2}, \quad\|B(s)\|_{\mathrm{HS}}<+\infty .
$$

Рассмотрим неслучайную функцию множеств $\mu_{\mathrm{U}}(\mathrm{A})=\sum_{i=1}^{\infty} \varphi_{i} \int_{\mathrm{A}} \sin i s d s$, где $\mu_{\mathrm{U}}-\mathrm{U}$-значная мера (это следует из аналога теоремы Никодима [15, теорема 8.6]). Мы можем взять $\mathrm{m}_{j}(d s)=d s$. Имеем

$\sum_{i=1}^{\infty} \int \mathrm{X}^{B}(s) \varphi_{i} d \mu_{i}(s)=\sum_{i=1}^{\infty} \sum_{j=1}^{\infty} \varphi_{j} \int_{[0, \pi]} \frac{1}{(i j)^{3 / 4}} \sin ^{2} i s d s=\frac{\pi}{2}\left(\sum_{j=1}^{\infty} \varphi_{j} \frac{1}{j^{3 / 4}}\right)\left(\sum_{i=1}^{\infty} \frac{1}{i^{3 / 4}}\right)$,

и последняя сумма расходится.

4.2. Эволюционные уравнения, ведомые U-значными СM. Пусть $\mu_{\mathrm{U}}$ определена на борелевской $\sigma$-алгебре в $\mathrm{X}=[0, T], Y_{0} \in \mathrm{H}$ и $B: \mathrm{U} \rightarrow \mathrm{H}$ - непрерывный линейный оператор. Мы рассмотрим мягкое решение уравнения (2)

$$
Y(t)=S(t) Y_{0}+\int_{(0, t]} S(t-s) B d \mu_{\mathrm{U}}(s), \quad 0 \leqslant t \leqslant T .
$$

У с л о в и е 5 . Для некоторого $\varepsilon>1 / 2$ оператор $A^{\varepsilon} B: \mathrm{U} \rightarrow \mathrm{H}$ является непрерывным линейным оператором Гильберта-Шмидта.

Теорема 4. Пусть выполняются условия 4 и 5. Тогда мягкое решение (20) корректно определено.

Д о к а з а т е ль с т в о. Мы проверим условия теоремы 3 для $\mathrm{A}=(0, t]$, $B(s)=S((t-s) \vee 0) B, 0 \leqslant s \leqslant T$. Для $0<\beta<2 \varepsilon-1$ в (17) получим

$$
\begin{gathered}
\sum_{i \geqslant 1} \sum_{n \geqslant 1} 2^{n \beta} \sum_{1 \leqslant k \leqslant 2^{n}}\left\|S\left(\left(t-d_{k n}\right) \vee 0\right) B \varphi_{i}-S\left(\left(t-d_{(k-1) n}\right) \vee 0\right) B \varphi_{i}\right\|^{2} \\
\stackrel{(13)}{\leqslant} C \sum_{n \geqslant 1} 2^{n \beta} \sum_{1 \leqslant k \leqslant 2^{n}} \sum_{i \geqslant 1} 2^{-2 n \varepsilon}\left\|A^{\varepsilon} B \varphi_{i}\right\|^{2} \leqslant C\left\|A^{\varepsilon} B\right\|_{\mathrm{HS}}^{2}<+\infty .
\end{gathered}
$$

Функция $B(s) \varphi_{i}$ непрерывна, поскольку $S(t)$ есть $C_{0}$-полугруппа. Теорема 4 доказана.

У с л о в и е 6 . Процесс $\mu_{\mathrm{U}}((0, s]), 0 \leqslant s \leqslant T$, имеет непрерывную модификацию.

Теорема 5. Пусть выполняются условия 4, 5 и 6. Тогда решение $Y(t)$ в (20) имеет непрерьвную модификацию.

Д о к а з а т е л ь с т в о. Имеем

$$
\int_{(0, t]} S(t-s) B d \mu_{\mathrm{U}}(s)=\int_{(0, T]} S((t-s) \vee 0) B d \mu_{\mathrm{U}}(s)-B \mu_{\mathrm{U}}((t, T]) .
$$

Процессы

$$
\eta^{(i)}(t)=\int_{(0, T]} S((t-s) \vee 0) B \varphi_{i} d \mu_{i}(s)
$$

непрерывны (см. доказательство непрерывности $\eta_{1}$ в теореме 2). Из оценок (18), (19) и (21) следует, что $\sum_{i=1}^{\infty} \eta^{(i)}(t)$ сходится равномерно по $t \in[0, T]$. Теорема 5 доказана.

\section{СПИСОК ЛИТЕРАТУРЫ}

1. Da Prato $G$. Some results on linear stochastic evolution equations in Hilbert spaces by the semigroups method. - Stoch. Anal. Appl., 1983, v. 1, p. 57-88.

2. Da Prato G., Zabczyk J. Stochastic Equations in Infinite Dimensions. Cambridge: Cambridge Univ. Press, 1992, 454 p. 
3. Peszat S., Zabczyk J. Stochastic partial differential equations with Lévy noise: an evolution equation approach. Cambridge: Cambridge Univ. Press, 2007, 419 p.

4. Duncan T.E., Pasik-Duncan B., Maslowski B. Fractional Brownian motion and stochastic equations in Hilbert spaces. - Stoch. Dyn., 2002, v. 2, № 2, p. 225-250.

5. Brzeźniak Z., van Neerven J. Stochastic convolution in separable Banach space and the stochastic linear Cauchy problem. - Studia Math., 2000, v. 143, № 1, p. 43-74.

6. van Neerven J.M.A.M., Weis L. Stochastic integration of functions with values in a Banach space. - Studia Math., 2005, v. 166, № 2, p. 131-170.

7. van Neerven J., Veraar M., Weis L. Stochastic evolution equations in UMD Banach spaces. - J. Funct. Anal., 2008, v. 255, № 4, p. 940-993.

8. Dettweiler J., Weis L., van Neerven J. Space-time regularity of solutions of the parabolic stochastic Cauchy problem. - Stoch. Anal. Appl., 2006, v. 24, № 4, p. 843869.

9. Mémin J., Mishura Yu., Valkeila E. Inequalities for the moments of Wiener integrals with respect to a fractional Brownian motion. - Statist. Probab. Lett., 2001, v. 51, № 2, p. 197-206.

10. Samorodnitsky G., Taqqu M.S. Stable Non-Gaussian Random Processes. Boca Raton: Chapman \& Hall, 1994, 632 p.

11. Kwapień S., Woycziński W. A. Random Series and Stochastic Integrals: Single and Multiple. Boston: Birkhäuser, 1992, 360 p.

12. Радченко В. Н. Интегралы по общим случайным мерам. Киев: Ин-т математики НАН Украины, 1999, 196 с. (Труды Института математики НАН Украины, т. 27.)

13. Радченко В. Н. Интегралы по случайным мерам и случайные линейные функционалы. - Теория вероятн. и ее примен., 1991, т. 36, в. 3, с. 594-596.

14. Радченко В.Н. Выборочные функции случайных мер и пространства Бесова. Теория вероятн. и ее примен., 2009, т. 54, в. 1, с. 158-166.

15. Drewnowski L. Topological rings of sets, continuous set functions, integration. III. Bull. Acad. Pol. Sci., 1972, v. 20, p. 439-445.

16. Вахания Н.Н., Тариеладзе В.И., Чобанян С. А. Вероятностные распределения в банаховых пространствах. М.: Наука, 1985, 368 с.

17. Radchenko V. M. Besov regularity of stochastic measures. - Statist. Probab. Lett., 2007 , v. 77 , № 8, p. 822-825.

18. Drewnowski $L$. Boundness of vector measures with values in the spaces $L_{0}$ of Bochner measurable functions. - Proc. Amer. Math. Soc., 1984, v. 91, p. 581-588.

19. Pazy A. Semigroups of Linear Operators and Applications to Partial Differential Equations. New York: Springer-Verlag, 1983, 280 p.

20. Kamont A. A discrete characterization of Besov spaces. - Approx. Theory Appl. (N.S.), 1997, v. 13, № 2, p. 63-77.

Поступила в редакцию 24.V.2013

Исправленный вариант 22.X.2013

(c) 2014 г.

СТАРЦЕВ А.Н.*

\title{
ОБ ОДНОМ ПОДХОДЕ К ОЦЕНКЕ ПАРАМЕТРОВ ДВУМЕРНОГО ПРОЦЕССА ЛИНЕЙНОЙ ДИФФУЗИИ В НЕСТАЦИОНАРНОМ СЛУЧАЕ
}

\begin{abstract}
Рассмотрены отличные от оценок максимального правдоподобия оценки параметров двумерного марковского гауссовского нестационарного процесса, заданного системой линейных стохастических уравнений с двумя параметрами. Доказана их асимптотическая нормальность и
\end{abstract}

* Институт математики им. В. И. Романовского АН РУз, Ташкент, Узбекистан; e-mail: anstartsev@gmail.com 